\title{
Fibrilación auricular e insuficiencia cardíaca
}

\author{
Dres. Gustavo Tortajada, Walter Reyes Caorsi, Gonzalo Varela
}

\begin{abstract}
Resumen mos fisiopatológicos.

Palabras clave:

\author{
FIBRILACIÓN ATRIAL \\ INSUFICIENCIA CARDÍACA \\ TRATAMIENTO FARMACOLÓGICO \\ TÉCNICAS DE ABLACIÓN
}

La fibrilación auricular es la arritmia clínica más frecuente y la que más comúnmente se asocia a la insuficiencia cardíaca, independientemente de la fracción de eyección del ventrículo izquierdo. Su aparición en pacientes con falla cardíaca y viceversa agrava la evolución clínica y aumenta la mortalidad. Ambas patologías comparten factores de riesgo y mecanis-

Esta revisión se concentra en la fibrilación auricular en la insuficiencia cardíaca con fracción de eyección reducida. Se analizan sus mecanismos y la interacción entre ambas patologías. Se presenta el manejo clínico actual de esta arritmia en este contexto, haciendo especial énfasis en la anticoagulación, pero considerando también las diferentes estrategias farmacológicas y no farmacológicas para el control de la frecuencia y el control del ritmo.

\section{Summary}

Atrial fibrillation is the most common clinical arrhythmia, and independently of left ventricular ejection fraction is commonly associated with heart failure. It appearance in patients with heart failure or vice versa worsen the clinical evolution and increases mortality. Both diseases share risk factors and physiopathologic mechanisms.

This review concentrates in atrial fibrillation in patients with heart failure and reduced left ventricular ejection fraction. Mechanisms and its interaction between both pathologies are analysed. Current clinical treatment of this arrhythmia in heart failure is presented, with emphasis in oral anticoagulation but also discussing the different pharmacological and non-pharmacological strategies to rate and rhythm control.

Key words: Atrial fibrillation; Heart failure; Drug therapy; Ablation techniques

Key words:

\author{
ATRIAL FIBRILLATION \\ HEART FAILURE \\ DRUG THERAPY \\ ABLATION TECHNIQUES
}

El envejecimiento de la población, el incremento de algunos factores de riesgo cardiovascular que comparten ambas patologías y la mejoría en los métodos de detección y diagnóstico han hecho que la realidad supere las proyecciones. La prevalencia de obesidad, por ejemplo, factor de riesgo para ambas patologías, se ha duplicado desde 1980 y predomina en los países de ingresos medios y bajos ${ }^{(4)}$.

Se estima un crecimiento sostenido de la prevalencia de la IC y de la FA en los próximos años que repercutirá significativamente en la calidad de vida de un número importante de personas a nivel mun-

Servicio de Electrofisiología. Centro Cardiovascular Casa de Galicia. Montevideo, Uruguay

Correspondencia: Dr. Gustavo Tortajada. Correo electrónico: seef@vera.com.uy

Recibido Set 27, 2017; aceptado Oct 3, 2017 


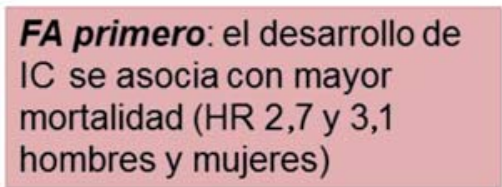

IC primero: el desarrollo de FA se asocia con mayor mortalidad (HR 1,6 y 2,7 hombres y mujeres)

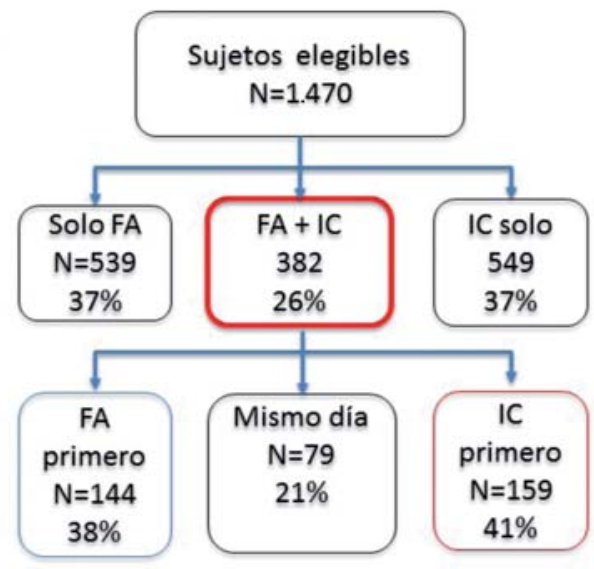

Figura 1. Relaciones temporales entre fibrilación auricular e insuficiencia cardíaca y su influencia en la mortalidad. Del estudio Framingham. Modificado de referencia ${ }^{(5)}$.

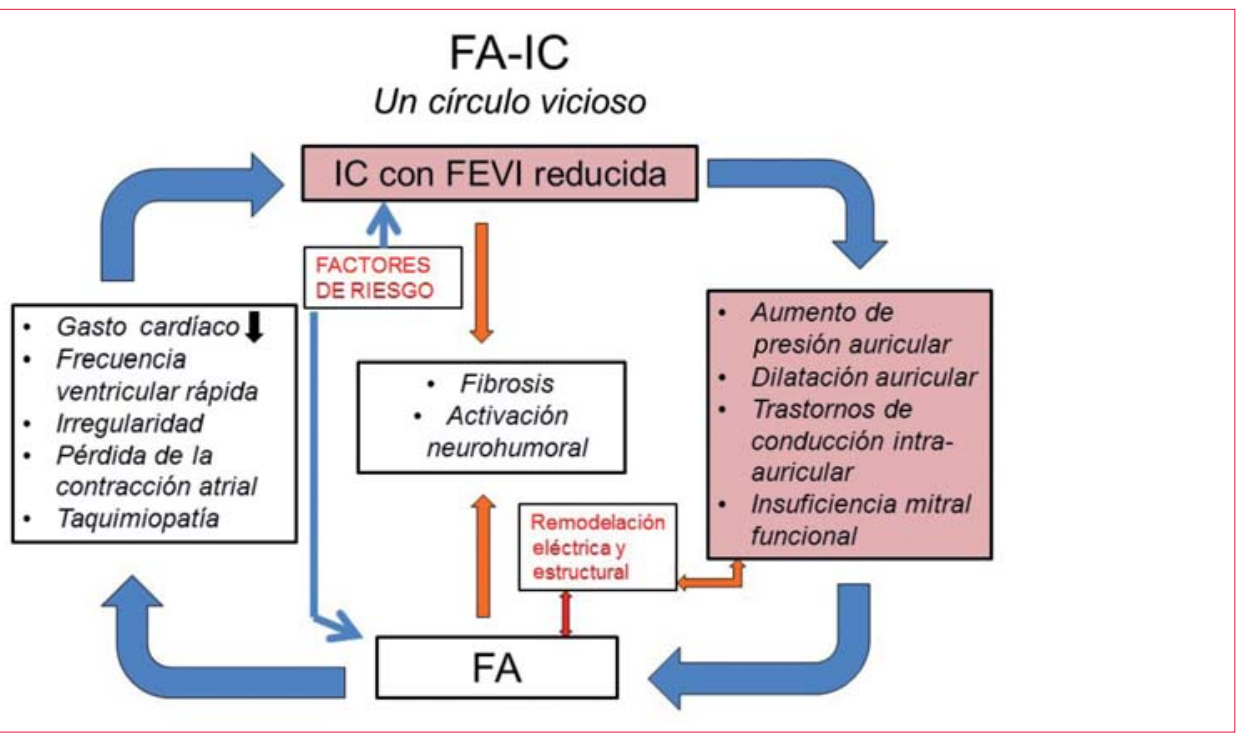

Figura 2. Relación entre los mecanismos de fibrilación auricular e insuficiencia cardíaca. Modificado de referencia (10).

dial y será un desafío para la sustentabilidad económica de los sistemas sanitarios.

La IC y la FA pueden ser causa o consecuencia una de la otra, y sus efectos deletéreos se potencian mutuamente: el desarrollo de IC en el paciente con FA aumenta la mortalidad de manera comparable a cuando la FA se instala en un paciente con $\mathrm{IC}^{(5)}$ (figura 1).

Ambas patologías comparten no solamente factores de riesgo, también una similar fisiopatología. Entre los mecanismos comunes se destacan el remodelado cardíaco estructural, la activación neurohormonal y la afectación del ventrículo izquierdo. En algunos casos la IC puede ser precipitada por la presencia de FA con frecuencia cardíaca elevada persistente (taquimiocardiopatía), siendo esta de mejor pronóstico que la FA asociada a miocardiopa- tías primarias. La FA de nueva aparición asociada a IC establecida tiene un peor pronóstico, seguramente vinculado a la presencia de una enfermedad miocárdica más evolucionada.

La IC, por otra parte, es el más fuerte predictor de la aparición de FA, multiplica en seis veces sus probabilidades $^{(6)}$.

En resumen, los pacientes con FA e IC tanto con fracción de eyección reducida (ICFEr) $(<40 \%)$ como preservada (ICFEp) (>50\%) tienen peor pronóstico, incluida una mayor mortalidad. La clasificación actual de la guía de IC de la Sociedad Europea de Cardiología introduce una nueva categoría de IC con FEVI descendida de rango intermedio (entre 40\%-49\%); los datos existentes en este grupo son es$\operatorname{casos}^{(7)}$. Nos referiremos específicamente a la FA en la ICFEr. 
2. Fibrilación auricular e insuficiencia cardíaca con fracción de eyección reducida

La FA y la ICFEr comparten, como se mencionó más arriba, múltiples factores de riesgo: diabetes, hipertensión, obesidad, presencia de cardiopatía (isquémica, valvular, miocardiopatía primaria). La comprensión de los mecanismos de ambas enfermedades y su relación podría generar estrategias para prevenir el desarrollo de las mismas.

La FA puede facilitar la aparición o la agravación de la IC por varios mecanismos (figura 2).

La presencia de una frecuencia cardíaca elevada genera un período de llenado diastólico reducido, con la consecuente reducción del gasto cardíaco. Además, el ritmo irregular compromete también el gasto cardíaco en aproximadamente un $25 \%$; los ciclos de llenado largo no logran compensar los ciclos cortos.

Los atrios que están destinados a fibrilar probablemente pierdan su función contráctil antes que se instale la FA.

Existen mecanismos capaces de compensar las alteraciones generadas por la recurrencia o permanencia de la FA, pero estos no se mantienen en el tiempo debido a la progresión paulatina de la fibrosis en las aurículas. El descenso del gasto cardíaco genera aumento de la epinefrina y endotelina incrementando el efecto vasoconstrictor propio de la $\mathrm{IC}^{(6,8)}$.

Cuando la frecuencia cardíaca está persistentemente elevada se incrementa la regurgitación mitral y se produce el consecuente aumento de la precarga del ventrículo izquierdo favoreciendo aún más la dilatación del anillo. Asimismo, en los pacientes con cardiomiopatía dilatada la FA puede causar por sí sola dilatación del anillo mitral con insuficiencia secundaria. La FA con frecuencia rápida mantenida puede ser, como mencionamos, causa primaria de deterioro de la función ventricular, siendo reversible con el control adecuado y oportuno de la frecuencia cardíaca o, como también ha sido demostrado, con el control del ritmo mediante una ablación exitosa de $\mathrm{FA}^{(9,10)}$.

La presencia de IC puede facilitar el desarrollo de FA: se generan cambios a nivel estructural, ultraestructural y neuroendócrino. A medida que evoluciona la disfunción ventricular con aumento de las presiones intracavitarias aumenta también el riesgo de desarrollo de FA al generarse cambios importantes a nivel auricular, agrandamiento con incremento de presión auricular e insuficiencia mitral funcional.

El volumen de la aurícula izquierda es uno de los parámetros ecocardiográficos de mayor valor predictivo de desarrollo y recurrencia de FA. Estas al- teraciones estructurales se acompañan de cambios eléctricos, cambios a nivel celular, anatómicos y ultraestructurales acumulativos; a nivel del sustrato, estos cambios pueden ser modulados con el uso de inhibidores de la angiotensina, antifibróticos y antiinflamatorios ${ }^{(10,11)}$.

La FA no solo es un marcador de IC avanzada, sino también un factor independiente de mortalidad y hospitalizaciones, aunque no todos los ensayos randomizados hayan podido demostrar esta relación. En el estudio V-HeFT la mortalidad de los pacientes con FA no era mayor que la de los pacientes en ritmo sinusal, pero esto podría explicarse porque los pacientes con FA enrolados presentaban una FEVI mayor que los no fibrilados ${ }^{(12)}$.

El estudio COMET analizó el valor pronóstico de la presencia de FA en una cohorte de pacientes con ICFEr tratados con betabloqueantes. Los autores encontraron una diferencia significativa con mayor mortalidad e internaciones por IC en los pacientes con FA. En el análisis multivariado observaron que la FA no es un factor de riesgo independiente de mortalidad al considerar el efecto de otros factores pronósticos de IC, si bien encontraron que la instalación de FA en la evolución es un fuerte predictor de muerte en los pacientes con IC ${ }^{(13)}$.

El ensayo MADIT II demostró que al ajustar los resultados por edad, clase funcional, función renal y el uso de betabloqueantes, la FA no se asoció a aumento de la mortalidad ${ }^{(14)}$.

En un estudio comunitario realizado entre 1986 y 2003, con el objetivo de determinar la mortalidad global en relación con la presencia de FA previa o posterior al desarrollo de IC, se analizaron 1.664 individuos con IC, 553 con historia de FA previa, 384 que desarrollan FA después de IC y 572 sin FA, con un seguimiento medio de cuatro años. El grupo con FA previa tuvo $29 \%$ de mayor mortalidad, pero el grupo con FA posterior tuvo el doble de mortalidad, ambos con respecto a los pacientes sin $\mathrm{FA}^{(15)}$.

Del análisis de estos ensayos surge el concepto de diferenciar a aquellos pacientes con FA establecida de aquellos que en el proceso de la enfermedad agregan FA. Más allá de que en ambos el pronóstico está comprometido, los pacientes con FA incidente (inicio reciente) tienen peor pronóstico, son frecuentemente de mayor edad y tienen una enfermedad cardíaca más avanzada.

La FA tiene un efecto negativo significativo en la mortalidad e internaciones de los pacientes con IC y FEVI reducida. 


\begin{tabular}{|l|l|}
\hline $\mathrm{CHA}_{2} \mathrm{DS}_{2}$-VASc & \\
\hline Insuficiencia cardíaca & 1 punto \\
\hline Diabetes mellitus & 1 punto \\
\hline Hipertensión arterial & 1 punto \\
\hline Edad $\geq 75$ años & 2 puntos \\
\hline ACV / AIT / TE & 2 puntos \\
\hline Enfermedad vascular (infarto, enf. arterial periférica, placa aórtica) & 1 punto \\
\hline Edad entre 65 y 74 años & 1 punto \\
\hline Sexo femenino & 1 punto \\
\hline SCORE MÁXIMO & 9 puntos \\
\hline
\end{tabular}

Figura 3. Score $\mathrm{CHA}_{2} \mathrm{DS}_{2} \mathrm{VASc}$, escala de riesgo de $\mathrm{ACV}$ en fibrilación auricular recomendada actualmente por todas las guías. ACV: accidente cerebrovascular; AIT: accidente isquémico transitorio; TE: tromboembolismo.

\section{Manejo de la fibrilación auricular en la insuficiencia cardíaca}

Llamativamente aún no se dispone de evidencia contundente y particular para el tratamiento de los pacientes con FA e ICFEr. Es conocido que estos suelen tener una respuesta más pobre a la terapéutica, suelen ser más viejos y tener más comorbilidades. Naturalmente, en el paciente con FA se mantienen las pautas terapéuticas determinadas para el manejo de la $\mathrm{IC}^{(6)}$.

El tratamiento óptimo de la ICFEr mediante el uso de inhibidores de la enzima convertidora de angiotensina o antagonistas de los receptores de angiontensina II, antagonistas de la aldosterona y betabloqueantes, reduce francamente la incidencia de FA en estos pacientes.

Por otra parte, y con respecto a los betabloqueantes (una de las piedras angulares en el tratamiento de la ICFEr), un metaanálisis demostró que no fueron tan útiles en pacientes con FA como lo son en pacientes que mantienen ritmo sinusal en la reducción de las hospitalizaciones por IC y de la mortalidad $^{(16)}$

El manejo de los factores higiénico-dietéticos tiene gran importancia en el desarrollo y progresión de la FA. Se ha demostrado una fuerte relación entre el sobrepeso y la obesidad en el desarrollo de todas las formas de FA. En el estudio Framigham se demostró un 50\% de aumento de la incidencia de FA en obesos. Se ha descrito también una relación entre la cantidad de grasa pericárdica y el desarrollo de $\mathrm{FA}^{(17,18)}$.

El descenso de peso no solo trae aparejado la disminución de la grasa pericárdica, sino también la reducción del tamaño auricular, mejoría en la función mecánica de la aurícula y mejoría del sustrato electrofisiológico. Como consecuencia se reduce el riesgo de desarrollo de FA y se disminuye la carga hemodinámica en los pacientes ya portadores de FA.

Aunque no ha sido aún valorada en un ensayo clínico randomizado, la hipótesis que plantea que la modificación de determinados factores de riesgo cardiovascular pueda disminuir el desarrollo de IC en pacientes con o sin FA, es muy atractiva.

Recientemente se publicaron resultados del Women's Health Study, que siguió durante más de 20 años a una población de 34.736 mujeres. El desarrollo de una nueva FA aumentaba significativamente el riesgo de desarrollar IC en la evolución (HR 9,03). Se identificaron además otros cuatro factores de riesgo de desarrollo de IC en esta población: hipertensión, obesidad, tabaquismo y diabetes. Su control adecuado podría prevenir el desarrollo de IC ${ }^{(19)}$.

Otros factores también se han relacionado fuertemente con la aparición de la arritmia además de la hipertensión arterial y la diabetes: la apnea del sueño y el exceso de ejercicio. El tratamiento con presión positiva continua en pacientes portadores de apnea del sueño posterior a la ablación de la FA disminuye la incidencia de recurrencias ${ }^{(20,21)}$.

El tratamiento y control adecuado de los factores de riesgo favorece el mantenimiento en ritmo sinusal.

\subsection{Anticoagulación}

La anticoagulación oral (ACO) es una parte esencial del tratamiento del paciente con FA para prevenir complicaciones embólicas. Al menos una tercera parte de los accidentes cerebrovasculares (ACV) isquémicos están vinculados a $\mathrm{FA}$; estos ACV son más graves y tienen mayor mortalidad que los no vinculados a $\mathrm{FA}^{(1,2)}$. 


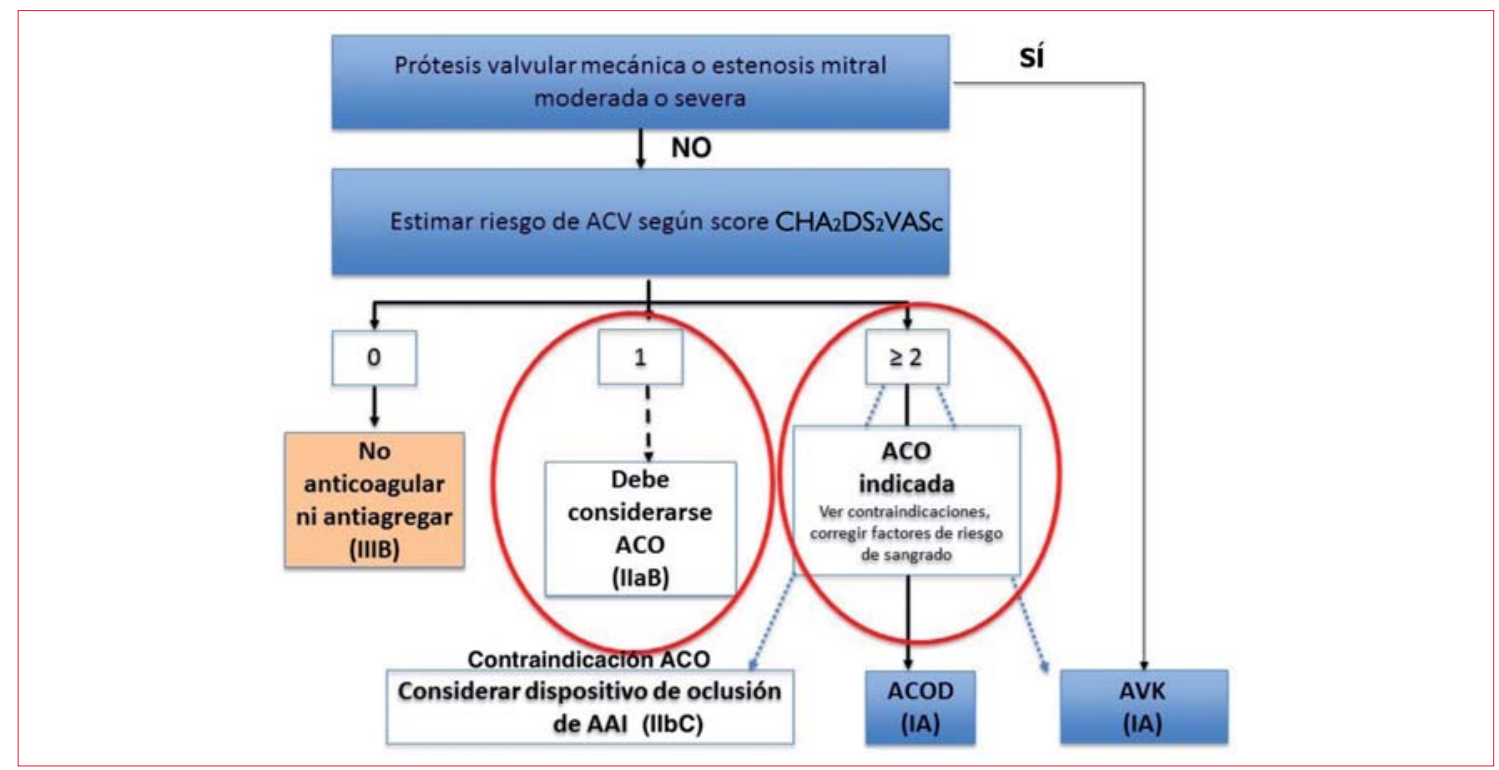

Figura 4. Modificado de referencia (1).

ACO: anticoagulación oral; AAI: apéndice auricular izquierdo; ACOD: anticoagulantes orales directos; AVK: anticoagulantes orales antagonistas de la vitamina K; ACV: accidente cerebrovascular.

Más allá de las implicancias clínicas en cuanto a morbimortalidad, este hecho tiene una enorme repercusión en los costos sanitarios ${ }^{(22)}$.

El tratamiento con ACO puede prevenir la mayoría de los ACV isquémicos en los pacientes con FA y de esa forma mejorar significativamente su pronóstico e impactar positivamente en los costos en salud. El tratamiento con ACO ha demostrado ser superior a la ausencia de tratamiento o al tratamiento con antiagregantes plaquetarios ${ }^{(1,2)}$.

Las guías europeas de FA recomiendan, para valorar el riesgo embólico en pacientes con FA y definir el uso de ACO, al score $\mathrm{CHA}_{2} \mathrm{DS}_{2} \mathrm{VASc}$ (figura 3), siendo hoy el score universalmente utilizado. Recomiendan el tratamiento con ACO a todos los pacientes con $\mathrm{CHA}_{2} \mathrm{DS}_{2} \mathrm{VASc} \geq 1$ (excepto cuando este punto se genere por el hecho de ser mujer) (figura 4). Concretamente, cuando el score es $\geq 2$ la recomendación del uso de ACO es IA, mientras que cuando el score es 1 el nivel de recomendación es IIaB, es decir, que debe considerarse, pues muchos pacientes podrían tener beneficio clínico de su utilización.

Por tanto, los pacientes con IC deberán recibir ACO de forma permanente y solo contraindicaciones reales o causas justificadas llevarán a la suspensión de la misma. Sin embargo, las guías norteamericanas publicadas dos años antes no obligan al tratamiento con $\mathrm{ACO}$ en pacientes con $\mathrm{CHA}_{2} \mathrm{DS}_{2} \mathrm{VASc}$ de $1^{(1,2)}$.

En el estudio ARISTOTELES (apixabán para reducción de ACV y otros fenómenos embólicos en pacientes con FA no valvular) se demostró que la incidencia de eventos embólicos era mayor en pacien- tes con ICFEr que en pacientes con ICFEp, y a su vez menos frecuentes en pacientes sin IC.

El tratamiento con apixabán redujo el número de eventos embólicos en comparación con la warfarina en los tres grupos ${ }^{(23)}$.

En un subanálisis de pacientes con síntomas de IC del estudio RELY (dabigatrán versus warfarina en pacientes con FA no valvular) se mostró que los eventos embólicos eran mayores en pacientes con historia de IC. Se demostró, además, una mayor eficacia en la prevención de embolias de dabigatrán frente a la warfarina para todos los pacientes. Sin embargo, un metaanálisis que comparó pacientes con FA e ICFEr versus ICFEp, si bien encontró que los que tenían compromiso de la FEVI tenían mayor mortalidad, el número de eventos embólicos fue similar ${ }^{(24,25)}$.

En todo paciente al cual se le indique ACO, se deberá evaluar el riesgo hemorrágico. La escala HASBLED es la más recomendada actualmente. Debe tenerse en cuenta que ningún valor en el score de riesgo hemorrágico contraindica el uso de ACO, aunque sí incidirá en los controles y precauciones, además de obligar a corregir los factores de riesgo hemorrágico modificables ${ }^{(1)}$.

Es útil recordar que el beneficio neto de la ACO para la prevención de embolias en los pacientes con FA es contundente, a pesar de lo cual sigue siendo un tratamiento subutilizado y con una frecuente suspensión prematura del mismo. Las complicaciones hemorrágicas, tanto graves como leves, el alto riesgo percibido de sangrado con la ACO y los esfuerzos necesarios para monitorizar y ajustar la do- 
sis de antagonistas de la vitamina $\mathrm{K}(\mathrm{AVK})$ son las razones más comunes para interrumpirlos o no prescribirlos $^{(26,27)}$.

Sin embargo, el riesgo de ACV sin ACO normalmente excede el riesgo de sangrado, incluso en pacientes de edad avanzada, pacientes con disfunción cognitiva o con frecuentes caídas o fragilidad ${ }^{(28,29)}$.

Debe recordarse que el riesgo de sangrado con ácido acetilsalicílico (AAS) es similar al del tratamiento con AVK o con anticoagulantes orales directos (ACOD) -dabigatrán, rivaroxabán y apixabán-, mientras que estos, pero no el AAS, previenen de manera eficaz los ACV en pacientes con $\mathrm{FA}^{(30,31)}$.

Los ACOD son los fármacos preferidos para el tratamiento de los pacientes con FA no valvular ya que, comparados con los AVK, parecen ser al menos igual de efectivos y más seguros (menos hemorragias intracraneales) tanto en aquellos pacientes con o sin IC. Sin embargo, persisten dudas sobre su seguridad en pacientes añosos y con función renal reducida, en los cuales se deberá ajustar la dosis.

No están indicados en pacientes en hemodiálisis y con aclaramiento de creatinina por debajo de 15 $\mathrm{ml} / \mathrm{minuto} / \mathrm{m}^{2}$.

Para pacientes con IC y FA que tienen válvulas cardíacas mecánicas o estenosis mitral moderada o severa, solo se deben emplear AVK para la prevención de las tromboembolias ${ }^{(1,2)}$.

Como alternativa a los ACO, en pacientes con contraindicación para esta medicación y riesgo embólico elevado, se puede considerar el uso de un dispositivo de oclusión de la orejuela auricular izquier$\mathrm{da}^{(32,33)}$.

La ACO, de preferencia con ACOD, es una parte esencial del tratamiento de todo paciente con FA $e$ $I C$.

\subsection{Control de la frecuencia ventricular}

A pesar de ser dos patologías de alta prevalencia existen aspectos de su manejo sobre los cuales no existe una evidencia categórica. Un ejemplo es la mejor estrategia para el control de la frecuencia ventricular; todavía no está definitivamente establecido si el control estricto de la frecuencia cardíaca es mejor que el control más laxo de la misma en los pacientes con FA e ICFEr.

El estudio RACE II randomizó pacientes con FA persistente a control laxo de frecuencia cardíaca versus control estricto de la misma, no habiéndose encontrado diferencias en la mortalidad. El riesgo de hemorragias, hospitalización por IC y la presencia de síntomas fueron similares en ambos grupos. Cabe destacar que dos tercios de los pacientes eran asintomáticos, y la diferencia de la frecuencia cardíaca entre ambos grupos fue de solo $10 \mathrm{pm}^{(34)}$.

Un análisis post hoc del RACE II, que se centró en pacientes con IC y FA, definió dos grupos, uno con ICFEr y otro con ICFEp. Aunque los pacientes con ICFEr eran un número pequeño, no existió diferencia entre ambos grupos (control estricto versus control laxo de la frecuencia) en mortalidad u hospitalizaciones, así como tampoco en los síntomas o en la calidad de vida.

En contraste, el registro sueco de IC demostró que en los pacientes con FA la mortalidad fue mayor en aquellos que presentaban una frecuencia cardíaca mayor de 100 pm y el uso de betabloqueantes se asoció a mejor pronóstico ${ }^{(35,36)}$.

Las guías norteamericanas (Colegio Americano de Cardiología - ACC, Asociación Americana del Corazón - AHA, Sociedad del Ritmo Cardíaco - HRS) de 2014 sobre manejo de la FA recomiendan un control laxo para pacientes asintomáticos y con función del ventrículo izquierdo conservada (recomendación clase IIb, nivel de evidencia B) ${ }^{(2)}$.

No existen estudios prospectivos que valoren específicamente el control de la frecuencia en pacientes con FA permanente e IC. En pacientes sintomáticos, el control adecuado de la frecuencia ventricular logra mejoría de la FEVI, que probablemente se deba a un componente taquimiocardiopático.

Por otra parte, la guía de IC 2016 de la Sociedad Europea de Cardiología considera que la frecuencia ventricular óptima en reposo en pacientes con FA e IC podría estar entre 60 y 100 pm, pero no está claramente establecido(6).

Los datos del RACE II son tomados también por la guía europea de FA del mismo año, recomendando una frecuencia menor de 110 pm como objetivo de tratamiento para el control de la frecuencia en reposo. Sin embargo, como se mencionó antes, el Grupo de Trabajo de la guía de IC considera que en pacientes con IC es preferible una frecuencia más baja $(60-100 \mathrm{pm})^{(1,6)}$.

Las frecuencias ventriculares $<70 \mathrm{pm}$ se han asociado a peores resultados. Esto podría explicar por qué los bloqueadores beta administrados en las dosis máximas recomendadas por las guías no lograron reducir la morbilidad o la mortalidad en pacientes con ICFEr y FA. Podría explicar también la asociación entre digoxina y los resultados adversos con esta droga comunicados en algunos estudios observacionales sobre $\mathrm{FA}^{(37-39)}$.

Resumiendo, en pacientes sintomáticos con mal control de frecuencia el control laxo parece inapropiado. De no lograrse un control adecuado de la frecuencia y de los síntomas con el tratamiento farmacológico o cuando existen efectos adversos intolera- 
bles con este, se deberá considerar la ablación del nodo auriculoventricular (AV) e implante de marcapaso definitivo.

Existe un grupo de pacientes con disfunción ventricular izquierda que responderá a la ablación del nodo AV e implante de marcapaso con estimulación unicameral desde el ventrículo derecho mejorando la función; sin embargo, otros pacientes pueden deteriorar su función con la estimulación desde el ápex del ventrículo derecho. El primer grupo, que mejora la función con la ablación y el control de la frecuencia, seguramente tenga asociado un componente taquimiocardiopático. Con la ablación del nodo AV se ha demostrado algún grado de taquimiocardiopatía en $25 \%$ a $50 \%$ de los pacientes ${ }^{(40,41)}$.

Un metaanálisis de Wood y colaboradores acerca de la evolución y sobrevida posterior a la ablación del nodo AV + marcapaso demostró una mejor tolerancia al ejercicio, una mejoría en la calidad de vida y también una mejoría en la FEVI en pacientes con disfunción previa. Sin embargo, no hubo, al año, diferencias signficativas con el tratamiento médico en cuanto a la sobrevida ${ }^{(42)}$.

Cabe recordar que la estimulación del ventrículo derecho puede tener consecuencias negativas en la función al generar disincronía ventricular, efectos adversos en el metabolismo y la perfusión con impacto en la remodelación del ventrículo izquierdo. Dado este potencial efecto deletéreo de la estimulación apical derecha, sumado al hecho de que el paciente se transforma en marcapaso dependiente, la estrategia de ablación del nodo AV e implante de marcapaso unicameral ha caído en descrédito, sin una clara justificación por la evidencia.

El advenimiento de la terapia de resincronización cardíaca (TRC) ha aportado otra herramienta útil en pacientes con bloqueo AV e ICFEr. La estimulación biventricular demostró ser mejor en comparación con la estimulación exclusiva del ventrículo derecho. Cuando se considera la TRC en pacientes con FA (pacientes con bloqueo de rama izquierda e ICFEr) se debe tener en cuenta tres factores: la TRC ha demostrado un beneficio en sobrevida, síntomas y parámetros ecocardiográficos comparables al de esta terapia en pacientes con ritmo sinusal; es imprescindible asegurar un porcentaje de estimulación cercano al $100 \%$; se debe considerar la ablación del nodo $\mathrm{AV}^{(43)}$.

Una revisión sistemática analizó 768 pacientes con FA + IC sometidos a TRC, 339 con ablación del nodo AV y los restantes con control farmacológico de la frecuencia. Se demostró que los pacientes con ablación del nodo AV tenían menor mortalidad global y cardiovascular, y una mejoría en la clase fun- cional en comparación con aquellos con control farmacológico de la frecuencia ventricular ${ }^{(44)}$.

También ha sido demostrado que la respuesta a la TRC + ablación AV es significativamente mejor en pacientes con miocardiopatia dilatada no isquémica que en la isquémica ${ }^{(45)}$.

Finalmente, debe recordarse que la TRC no ha demostrado disminuir la carga de FA en pacientes con ICFEr, aunque algunos pacientes resincronizados pueden recuperar ritmo sinusal ${ }^{(43)}$.

\subsection{Control del ritmo}

3.3.1. Prevención de fibrilación auricular en pacientes con disfunción ventricular

Tanto las guías norteamericanas como las europeas más recientes sugieren el tratamiento con inhibidores de la enzima convertidora de la angiotensina o inhibidores de la angiotensina II y de betabloqueantes en los pacientes con disfunción ventricular para prevención primaria de FA. También el uso de eplerenona ha demostrado su utilidad con este objetivo. No se recomiendan para prevención secundaria $^{(1,2,46)}$.

\subsubsection{Control farmacológico del ritmo}

Las guías europeas 2016 recomiendan el control del ritmo para la mejoría sintomática en los pacientes con FA (recomendación clase I) ${ }^{(1)}$.

Muchas veces es difícil valorar clínicamente en forma adecuada la repercusión sintomática de la FA. El paciente se adapta a sus limitaciones y puede no percibir o transmitir adecuadamente las molestias. La reversión a ritmo sinusal con una cardioversión eléctrica y el uso de drogas antiarrítmicas para mantener, aunque sea transitoriamente, el ritmo sinusal, es de gran ayuda en discriminar la real repercusión sintomática de la FA.

En los pacientes con disfunción ventricular izquierda los recursos farmacológicos disponibles para el mantenimiento del ritmo sinusal son limitados. Según la evidencia actual las únicas drogas aceptables para estos pacientes son dofetilide (no disponible en nuestro país) y amiodarona. Son bien conocidas las limitaciones de la amiodarona, sobre todo para su uso en el mediano y largo plazo, por lo cual de elegirse este camino y ser efectiva en mantener el ritmo sinusal, debe usarse la mínima dosis posible ${ }^{(10)}$.

No existe evidencia de que el control del ritmo con amiodarona mejore significativamente el pronóstico de estos pacientes. El ensayo clínico más importante que comparó una estrategia de control de la frecuencia versus control del ritmo en pacientes con FA e IC fue el AF-CHF. En este estudio se ran- 
domizaron 1.376 pacientes a una estrategia de control del ritmo o de control de la frecuencia. En ambos grupos, $80 \%$ de los pacientes recibían betabloqueantes. En la rama de control del ritmo, 80\% fueron tratados con amiodarona, y estaba en ritmo sinusal el $73 \%$ al final de 47 meses de seguimiento; en la rama de control de la frecuencia solo $35 \%$ estaban con ritmo sinusal. Sin embargo, la mortalidad cardiovascular, objetivo primario del estudio, fue similar en ambos grupos. Además, las internaciones fueron mayores en el grupo de control del ritmo ${ }^{(47)}$.

El control farmacológico del ritmo no ha demostrado mejorar el pronóstico de los pacientes con FA.

\subsubsection{Ablación de la fibrilación auricular}

A pesar del tratamiento farmacológico el desarrollo de IC en pacientes con FA no ha descendido en las últimas décadas, confirmando sus limitaciones ${ }^{(48)}$.

Más allá del énfasis en el valor del control de sus factores de riesgo, la ablación de la FA ha surgido y se ha posicionado como una alternativa muy atractiva al tratamiento farmacológico, con resultados mejores a este ${ }^{(2)}$.

Se han publicado numerosos estudios observacionales de ablación de FA en pacientes con IC que fueron a su vez analizados en conjunto en varios metaanálisis. Estos evidenciaron un éxito del procedimiento variable entre $54 \%$ y $82 \%$ luego de más de un procedimiento, una tasa de éxito menor a la de otras poblaciones ${ }^{(10,49)}$.

Recientemente se publicó un ensayo clínico randomizado, AATAC, comparando en un grupo de pacientes con FA e ICFEr (clase funcional II-III) portadores de un dispositivo (cardiodesfibrilador con o sin resincronización ventricular) el tratamiento con amiodarona (101 pacientes) versus la ablación (102 pacientes). El punto final primario fue la recurrencia de FA, que en este caso pudo ser valorada con gran precisión debido al dispositivo. Luego de un seguimiento de 24 meses, $70 \%$ de los pacientes del grupo ablación no tuvieron recurrencias de FA luego de $1,4 \pm 0,6$ procedimientos versus $34 \%$ en el grupo amiodarona ( $\mathrm{p}<0,001)$. También se demostró una menor tasa de hospitalizaciones y una menor mortalidad, puntos finales secundarios, de menor peso pues el número de eventos fue bajo. La tasa de éxito del primer procedimiento de ablación fue variable en los diferentes centros entre $29 \%$ a $61 \%(50)$

En el estudio controlado PABA-CHF fue comparada la ablación de FA, en particular el aislamiento eléctrico de las venas pulmonares (41 pacientes), con la ablación del nodo AV + implante de marcapaso o desfibrilador con estimulación biventricular (40 pacientes) en pacientes con FA refractaria al
Resumen conceptual

- La IC es el más fuerte predictor de la aparición de FA.

- La FA puede facilitar la aparición o la agravación de la IC.

- La FA tiene un efecto negativo significativo en la mortalidad e internaciones de los pacientes con ICFEr.

- El tratamiento y control adecuado de los factores de riesgo favorece el mantenimiento en ritmo sinusal.

- La ACO es una parte esencial del tratamiento de todo paciente con FA e IC.

- En pacientes sintomáticos con mal control de frecuencia el control laxo parece inapropiado.

- El control farmacológico del ritmo no ha demostrado mejorar el pronóstico de los pacientes con FA.

- La ablación de FA en pacientes con IC es una estrategia a tener en cuenta.

tratamiento farmacológico. Los pacientes del grupo ablación de la FA mejoraron significativamente su calidad de vida, el test de caminata de 6 minutos y mejoraron la FEVI. El seguimiento fue particularmente corto, solo de seis meses ${ }^{(51)}$.

El seguimiento a más largo plazo (46 meses) en pacientes con FAy disfunción ventricular izquierda sometidos a ablación mostró una mayor tasa de recurrencias, aunque aquellos que se mantenían en ritmo sinusal demostraban una gran mejoría. Esto genera una gran expectativa en cuanto a que la evolución de las técnicas de ablación y accesorias y la mayor experiencia de los grupos puedan optimizar estos resultados ${ }^{(52)}$.

Técnicas de imagen podrían también ayudar a discriminar a priori quiénes son los pacientes que tienen más probabilidades de mejorar con este procedimiento y precisar la indicación. Un estudio con resonancia nuclear magnética evidenció que aquellos pacientes sin realce con gadolinio (ausencia de fibrosis en ventrículo izquierdo) tienen mayor probabilidad de mejoría con la ablación de la $\mathrm{FA}^{(53)}$.

La ablación de FA en pacientes con IC es una estrategia a tener en cuenta. Sin embargo, debemos considerar que es más factible que los pacientes requieran más de un procedimiento y que los resultados no son aún comparables a los obtenidos en los pacientes sin cardiopatía.

\section{Conclusiones}

Prevenir complicaciones y mantener una buena calidad de vida son los objetivos del tratamiento. La estra- 
tegia general para el tratamiento de la FA no varía entre los pacientes con IC y otros, pero conviene jerarquizar el control estricto de ciertos factores de riesgo. Debemos recordar que el único tratamiento con un valor pronóstico probado en estos pacientes es el adecuado uso de la anticoagulación para prevención del ACV. El tratamiento intervencionista es un recurso a considerar en ciertos pacientes.

\section{Bibliografía}

1. Kirchof P, Benussi S, Kotecha D, Ahlsson A, Atar D, Casadei B, et al. 2016 ESC Guidelines for the management of atrial fibrillation developed in collaboration with EACTS. Eur Heart J. 2016; 37(38): 2893-2962. doi: 10.1093/eurheartj/ehw210.

2. January CT, Wann LS, Alpert JS, Calkins H, Cigarroa JE, Cleveland JC, et al. 2014 AHA/ACC/HRS Guideline for the management of patients with atrial fibrillation: a report of the American College of Cardiology/American Heart Association Task Force on Practice Guidelines and the Heart Rhythm Society. Circulation. 2014; 130(23):2071-104. doi: 10.1161/CIR.0000000000000040.

3. Braunwald E. Shattuck lecture-cardiovascular medicine at the turn of the millennium: triumphs, concerns, and opportunities. N Engl J Med. 1997; 337(19):1360-9. doi: 10.1056/NEJM199711063371906.

4. Organización Mundial de la Salud. Obesidad y sobrepeso. Nota descriptiva No. 311. Octubre 2017. Disponible en: http://www.who.int/mediacentre/factsheets/fs311/es/.

5. Wang TJ, Larson MG, Levy D, Vasan RS, Leip EP, Woff PA, et al. Temporal relations of atrial fibrillation and congestive heart failure and their joint influence on mortality. The Framingham Heart Study. Circulation. 2003; 107:2920-2925. doi: 10.1161/01.CIR.0000072767.89944.6E.

6. Grönefeld GC, Hohnloser SH. Heart failure complicated by atrial fibrillation: mechanistic, prognostic, and therapeutic implications. J Cardiovasc Pharmacol Ther. 2003; 8(2):107-13 doi: $10.1177 / 107424840300800203$.

7. Ponikowski P, Voors AA, Anker SD, Bueno H, Cleland JGF, Coats AJS, et al. 2016 ESC Guidelines for the diagnosis and treatment of acute and chronic heart failure: The task force for the diagnostic and treatment of acute and chronic heart failure of the European Society of Cardiology (ESC) Developed with the special contribution of the Heart Failure Association (HFA) of the ESC. Eur Heart J. 2016; 37(27):2129-200. Advanced access published May 20, 2016. doi: 10.1093/eurheartj/ehw128.

8. Wasmund SL, Li JM, Page RL, Joglar JA, Kowal RC, Smith ML, et al. Effect of atrial fibrillation and an irregular ventricular response on sympathetic nerve activity in human subjects. Circulation. 2003; 107:2011-15. doi: 10.1161/01.CIR.0000064900.76674.CC.

9. Hsu LF, Jaïs $\mathbf{P}$, Sanders $\mathbf{P}$, Garrigue $\mathbf{S}$, Hocini M, Sacher F, et al. Catheter ablation for atrial fibrillation in congestive heart failure. N Engl J Med. 2004; 351:2373-83. doi: 10.1056/NEJMoa041018.

10. Verma A, Kalman JM, Callans DJ. Treatment of patients with atrial fibrillation and heart failure with reduced ejection fraction. Circulation. 2017; 135(16):1547-63. doi: 10.1161/CIRCULATIONAHA.116.026054.

11. Shi Y, Li D, Tardif JC, Nattel S. Enalapril effects on atrial remodeling and atrial fibrillation in experimental congestive heart failure. Cardiovasc Res. 2002; 54(2):456-61. doi: 10.1016/S0008-6363(02)00243-2.

12. Carson PE, Johnson GR, Dunkman WB, Fletcher RD, Farrell L, Cohn JN. The influence of atrial fibrillation on prognosis in mild to moderate heart failure. The V-HeFT studies. The V-HeFT VA Cooperative Studies Group. Circulation. 1993; 87(6 Suppl):VI102-10.

13. Poole-Wilson PA, Swedberg K, Cleland JGF, Di Lenarda A, Hanrath P,Komajda M, et al. Comparison of carvedilol and metoprolol on clinical outcomes in patients with cronic heart failure in the Carvedilol or Metoprolol European Trial (COMET): randomised controlled trial. Lancet. 2003; 362(9377):7-13. doi: 10.1016/S0140-6736(03)13800-7.

14. Zareba W, Steinberg JS, McNitt S, Daubert JP, Piotrowicz K, Moss AJ; MADIT II Investigators. Implantable cardioverter-defibrillator therapy and risk of congestive heart failure or death in MADIT II patients with atrial fibrillation. Heart Rhythm 2006; 3(6):631-7. doi: 10.1016/j.hrthm.2006.02.012.

15. Chamberlain AM, Redfield MM, Alonso A, Weston SA, Roger VL. Atrial fibrillation and mortality in heart failure: a community study. Circ Heart Fail 2011; 4(6):740-6. doi: 10.1161/CIRCHEARTFAILURE.111.962688.

16. Rienstra M, Damman K, Mulder BA, Van Gelder IC, McMurray JJV, Van Veldhuisen DJ. Beta-blockers and outcome in heart failure and atrial fibrillation: a meta-analysis. JACC Heart Fail 2013; 1(1):21-8. doi: 10.1016/j.jchf.2012.09.002.

17. Wang TJ, Parise H, Levy D, D'Agostino RB, Wolf PA, Vasan RS, et al. Obesity and the risk of new-onset atrial fibrillation. JAMA 2004; 292(20):2471-7. doi: 10.1001/jama.292.20.247.

18. Thanassoulis G, Massaro JM, O'Donnell CJ, Hoffmann U, Levy D, Ellinor PT, et al. Pericardial fat is associated with prevalent atrial fibrillation: the Framingham Heart Study. Circ Arrhythm Elec- 
trophysiol 2010; 3(4):345-50.

doi: 10.1161/CIRCEP.109.912055.

19. Chatterjee NA, Chae CU, Kim E, Vinayaga Moorthy MV, Conen D, Sandhu RK, et al. Modifiable risk factors for incident heart failure in atrial fibrillation. J Am Col Cardiol Heart Fail 2017; 5(8):552-60. doi: 10.1016/j.jchf.2017.04.004. Mayo 31. Epub ahead of print.

20. Schaer BA, Schneider C, Jick SS, Conen D, Osswald S, Meier CR. Risk for incident atrial fibrillation in patients who receive antihypertensive drugs: a nested case-control study. Ann Intern Med $2010 ; 152(2): 78-84$

21. Li L, Wang ZW, Li J, Ge X, Guo LZ, Wang Y, et al. Efficacy of catheter ablation of atrial fibrillation in patients with obstructive sleep apnea with and without continous positive airway pressure treatment: a meta-analysis of observational studies. Europace 2014; 16(9):1309-14. doi: 10.1093/europace/euu066.

22. Li X, Tse VC, Au Doung LW, Wong ICK, Chan EW. The impact of ischemic stroke on atrial fibrillation-related healthcare cost: A systematic review. Europace 2017; 19(6):937-47. doi: 10.1093/europace/euw093. Advance access published May 21, 2016.

23. McMurray JJ, Ezekowitz JA, Lewis BS, Gersh BJ, van Diepen S, Amerena J, et al. Left ventricular systolic dysfunction, heart failure, and the risk of stroke and systemic embolism in patients with atrial fibrillation: insights from the ARISTOTLE trial. Circ Heart Fail 2013; 6(3):451-60. doi: 10.1161/CIRCHEARTFAILURE.112.000143.

24. Ferreira J, Ezekowitz MD, Connolly SJ, Brueckmann M, Fraessdorf M, Reilly PA, et al; RE-LY Investigators. Dabigatran compared with warfarin in patients with atrial fibrillation and symptomatic heart failure: a subgroup analysis of the RE-LY trial. Eur J Heart Fail 2013; 15(9):1053-61. doi: 10.1093/eurjhf/hft111.

25. Kotecha D, Chudasama R, Lane DA, Kirchhof P, Lip GY. Atrial fibrillation and heart failure due to reduced versus preserved ejection fraction: A systematic review and meta-analysis of death and adverse outcomes. Int J Cardiol 2016; 203:660-6. doi 10.1016/j.ijcard.2015.10.220.

26. Le Heuzey JY, Ammentorp B, Darius H, De Caterina R, Schilling RJ, Schmitt J, et al. Differences among western European countries in anticoagulation management of atrial fibrillation. Data from the PREFER IN AF registry. Thromb Haemost 2014 111(5):833-41.

27. O'Brien EC, Holmes DN, Ansell JE, Allen LA, Hylek E, Kowey PR, et al. Physician practices regarding contraindications to oral anticoagulation in atrial fibrillation: findings from the Outcomes Registry for Better Informed Treatment of Atrial Fibri- llation (ORBIT-AF) registry. Am Heart J 2014; 167(4):601-9. doi: 10.1016/j.ahj.2013.12.014.

28. Donze J, Clair C, Hug B, Rodondi N, Waeber G, Cornuz J, et al. Risk of falls and major bleeds in patients on oral anticoagulation therapy. Am J Med 2012 ; $125(8): 773-8$.

29. Man-Son-Hing M, Nichol G, Lau A, Laupacis A. Choosing antithrombotic therapy for elderly patients with atrial fibrillation who are at risk for falls. Arch Intern Med 1999; 159(7):677-85. doi: 10.1001/archinte.159.7.677.

30. Mant J, Hobbs FDR, Fletcher K, Roalfe A, Fitzmaurice D, Lip GYH, et al; BAFTA investigators; Midland Research Practices Network (MidReC). Warfarin versus aspirin for stroke prevention in an elderly community population with atrial fibrillation (the Birmingham Atrial Fibrillation Treatment of the Aged Study, BAFTA): a randomized controlled trial. Lancet 2007; 370(9586):493-503. doi: 10.1016/S0140-6736(07)61233-1.

31. Diener HC, Eikelboom J, Connolly SJ, Joyner CD, Hart RG, Lip GY, et al; AVERROES Steering Committee and Investigators. Apixaban versus aspirin in patients with atrial fibrillation and previous stroke or transient ischaemic attack: a predefined subgroup analysis from AVERROES, a randomized trial. Lancet Neurol 2012; 11(3):225-31. doi: 10.1016/S1474-4422(12)70017-0.

32. Holmes DR, Doshi SK, Kar S, Price MJ, Sanchez JM, Sievert H, et al. Left atrial appendage closure as an alternative to warfarin for stroke prevention in atrial fibrillation: a patient-level meta-analysis. J Am Coll Cardiol 2015; 65(24):2614-23. doi: 10.1016/j.jacc.2015.04.025.

33. Price MJ, Reddy VY, Valderrabano M, Halperin JL, Gibson DN, Gordon N, et al. Bleeding outcomes after left atrial appendage closure compared with long-term warfarin: a pooled, patient-level analysis of the WATCHMAN randomized trial experience. JACC Cardiovasc Interv 2015; 8(15):1925-32. doi: 10.1016/j.jcin.2015.08.035.

34. Van Gelder IC, Groenveld HF, Crijns HJGM, Tuininga YS, Tijssen JGP, Alings AM, et al; RACE II Investigators. Lenient versus strict rate control in patients with atrial fibrillation. N Engl $J$ Med 2010; 362:1363-73.

35. Mulder BA, Van Veldhuisen DJ, Crijns HJGM, Tijssen JGP, Hillege HL, Alings M, et al; RACE II Investigators. Lenient vs. strict rate control in patients with atrial fibrillation and heart failure: a post-hoc analysis of the RACE II study. Eur J Heart Fail 2013; 15(11):1311-8. doi: 10.1093/eurjhf/hft093.

36. Li SJ, Sartipy U, Lund LH, Dahlström U, Adiels M, Petzold M, et al. Prognostic significance of resting heart rate and use of $\beta$-blockers in atrial fibrillation and sinus rhythm in patients with heart failure and redu- 
ced ejection fraction: findings from the Swedish Heart Failure Registry. Circ Heart Fail 2015; 8(5):871-9. doi: 10.1161/CIRCHEARTFAILURE.115.002285.

37. Allen LA, Fonarow GC, Simon DN, Thomas LE, Marzec LN, Pokorney SD, et al. Digoxin use and subsequent outcomes among patients in a contemporary atrial fibrillation cohort. J Am Coll Cardiol 2015; 65(25):2691-8. doi: 10.1016/j.jacc.2015.04.045.

38. Gheorghiade M, Fonarow GC, van Veldhuisen DJ, Cleland JGF, Butler J, Epstein AE, et al. Lack of evidence of increased mortality among patients with atrial fibrillation taking digoxin: findings from post hoc propensity-matched analysis of the AFFIRM trial. Eur Heart J 2013; 34(20):1489-97. doi: 10.1093/eurheartj/eht120.

39. Turakhia MP, Santangeli P, Winkelmayer WC Xu X, Ullal AJ, Than CT, et al. Increased mortality associated with digoxin in contemporary patients with atrial fibrillation: Findings From the TREAT-AF Study. J Am Coll Cardiol 2014; 64(7):660-8. doi: 10.1016/j.jacc.2014.03.060.

40. Rodriguez LM, Smeets JLRM, Xie B, de Chillou C, Cheriex E, Pieters F, et al. Improvement in left ventricular function by ablation of atrioventricular nodal conduction in selected patients with lone atrial fibrillation. Am J Cardiol 1993; 72(15):1137-41.

41. Redfield MM, Kay GN, Jenkins LS, Mianulli M,Jensen DN, Ellengogen KA, for the APT investigators. Tachycardia-related cardiomyopathy: A common cause of ventricular dysfunction in patients with atrial fibrillation referred for atrioventricular ablation. Mayo Clinic Proc 2000;75:790-5. doi: 10.1016/0002-9149(93)90982-I.

42. Wood MA, Brown-Mahoney C, Kay GN, Ellenbogen KA. Clinical outcomes after ablation and pacing therapy for atrial fibrillation: a meta-analysis. Circulation. $2000 ; 101(10): 1138-44$.

43. Daubert JC, Saxon L, Adamson PB, Auricchio A, Berger RD, Beshai JF, et al. 2012 EHRA/HRS expert consensus statement on cardiac resynchronization therapy in heart failure: implant and follow-up recommendations and management. Heart Rhythm 2012; 9(9):1524-76. doi: 10.1016/j.hrthm.2012.07.025.

44. Ganesan AN, Brooks AG, Roberts-Thomson KC, Lau DH, Kalman JM, Sanders P. Role of AV nodal ablation in cardiac resynchronization in patients with coexistent atrial fibrillation and heart failure: a systematic review. J Am Coll Cardiol 2012; 59(8):719-26. doi:10.1016/j.jacc.2011.10.891.

45. Sohinki D, Ho, J, Srinivasan N, Collins LJ, Obel OA. Outcomes after atrioventricular node ablation and biventricular pacing in patients with refractory atrial fibrillation and heart failure: a comparison between non-ischaemic and ischaemic cardiomyopathy. Europace 2014; 16(6):880-6. doi: 10.1093/europace/eut392.

46. Reyes Caorsi W. Tratamiento farmacológico no tradicional. En: Gonzalez Zuelgaray J. Tratamiento de la fibrilacion auricular. Buenos Aires: Inter-Médica; 2009. Capítulo 6. Pag 56-68.

47. Roy D, Talajic M, Nattel S, Wyse G, Dorian P, Lee KL, et al. Rhythm Control versus Rate Control for Atrial Fibrillation and Heart Failure. New Engl J Med 2008; 358(25):2667-77.

48. Chamberlain AM, Gersh BJ, Alonso A, Kopecky SL, Killian JM, Weston SA, et al. No decline in the risk of heart failure after incident atrial fibrillation: A community study assessing trends overall and by ejection fraction. Heart Rhythm 2017:14:791-798. doi: 10.1016/j.hrthm.2017.01.031.

49. Anselmino M, Matta M, D'Ascenzo F, Bunch TJ, Schilling RJ,Hunter RJ, et al. Catheter ablation of atrial fibrillation in patients with left ventricular dysfunction: a systematic review and meta-analysis. Circ Arrhythm Electrophysiol 2014; 7(6):1011-8. doi: 10.1161/CIRCEP.114.001938.

50. Di Biase L, Mohanty P, Mohanty S, Santngeli P, Trivedi C, Lakkireddy D, et al. Ablation versus Amiodarone for treatment of persistent Atrial Fibrillation in patients with congestive heart failure and an implanted device: results from the AATAC Multicenter Randomized Trial. Circulation 2016; 133(17):1637-44 doi: 10.1161/CIRCULATIONAHA.115.019406.

51. Khan MN, Jaïs P, Cummings J, Di Biase L, Sanders P, Martin DO, et al. PABA-CHF Investigators. Pulmonary-vein isolation for atrial fibrillation in patients with heart failure. N Engl J Med. 2008; 359:1778-85. doi: 10.1056/NEJMoa0708234.

52. Anselmino M, Grossi S, Scaglione M, Castagno D, Bianchi F, Senatore G, et al. Long-term results of transcatheter atrial fibrillation ablation in patients with impaired left ventricular systolic function. J Cardiovasc Electrophysiol 2013; 24(1):24-32. doi: 10.1111/j.1540-8167.2012.02419.x.

53. Ling LH, Taylor AJ, Ellims AH, Iles LM, McLellan AJ, Lee G, et al. Sinus rhythm restores ventricular function in patients with cardiomyopathy and no late gadolinium enhancement on cardiac magnetic resonance imaging who undergo catheter ablation for atrial fibrillation. Heart Rhythm 2013; 10(9):1334-9. doi: 10.1016/j.hrthm.2013.06.019. 